\title{
OPEN Altered TGFB1 regulated pathways promote accelerated tendon healing in the superhealer MRL/ MpJ mouse
}

Jacob G. Kallenbach ${ }^{1,2,6}$, Margaret A. T. Freeberg ${ }^{1,2,6}$, David Abplanalp ${ }^{1,2}$, Rahul G. Alenchery ${ }^{1,2}$, Raquel E. Ajalik ${ }^{1,2}$, Samantha Muscat ${ }^{2}$, Jacquelyn A. Myers ${ }^{4}$, John M. Ashton ${ }^{4}$, Alayna Loiselle ${ }^{2,3}$, Mark R. Buckley ${ }^{1,2}$, Andre J. van Wijnen ${ }^{5}$ \& Hani A. Awad ${ }^{1,2,3 凶}$

To better understand the molecular mechanisms of tendon healing, we investigated the Murphy Roth's Large (MRL) mouse, which is considered a model of mammalian tissue regeneration. We show that compared to $\mathrm{C} 57 \mathrm{BI} / 6 \mathrm{~J}$ (C57) mice, injured MRL tendons have reduced fibrotic adhesions and cellular proliferation, with accelerated improvements in biomechanical properties. RNA-seq analysis revealed that differentially expressed genes in the $\mathrm{C} 57$ healing tendon at 7 days post injury were functionally linked to fibrosis, immune system signaling and extracellular matrix (ECM) organization, while the differentially expressed genes in the MRL injured tendon were dominated by cell cycle pathways. These gene expression changes were associated with increased $\alpha-S M A+$ myofibroblast and F4/80+ macrophage activation and abundant BCL-2 expression in the C57 injured tendons. Transcriptional analysis of upstream regulators using Ingenuity Pathway Analysis showed positive enrichment of TGFB1 in both C57 and MRL healing tendons, but with different downstream transcriptional effects. MRL tendons exhibited of cell cycle regulatory genes, with negative enrichment of the cell senescence-related regulators, compared to the positively-enriched inflammatory and fibrotic (ECM organization) pathways in the $\mathrm{C} 57$ tendons. Serum cytokine analysis revealed decreased levels of circulating senescence-associated circulatory proteins in response to injury in the MRL mice compared to the C57 mice. These data collectively demonstrate altered TGFB1 regulated inflammatory, fibrosis, and cell cycle pathways in flexor tendon repair in MRL mice, and could give cues to improved tendon healing.

Flexor tendons transmit the intricate muscle forces required to move the digits freely. These hierarchically structured and tightly aligned collagen-rich connective tissues are sparsely populated and maintained by tenocytes, which are fibroblast-like cells that express phenotypic transcriptional factors including scleraxis $(\mathrm{Scx})^{1,2}$, mohawk $(\mathrm{Mkx})^{3,4}$, the tendon matrix protein of collagen type I (Col1), and non-collagenous proteins such as the glycoprotein tenomodulin (Tnmd) ${ }^{5,6}$. Injuries to flexor tendons, particularly in zone II of the hand, are susceptible to debilitating fibrotic adhesions, which compromise tendon gliding, strength, and range of motion, which is often attributed to the tendon's sparse cellularity ${ }^{7}$ and vascularity ${ }^{8}$. Upon injury, tendon healing proceeds in three canonical and overlapping phases: (1) an inflammation phase, (2) a proliferative, reparative phase, and (3) a remodeling phase $\mathrm{e}^{9,10}$. Immediately following tendon injury, acute cytokine and chemokine production signals circulating and peripheral inflammatory cells, predominantly leukocytes, to the hematoma forming at the injury site. The proliferative, reparative phase is driven by intrinsically and extrinsically derived fibroblasts, including the tenocytes, fibroblasts from the loose connective tissues enveloping the tendon (epitenon or paratenon) and its collagenous fascicles (endotenon) and perivascular cells ${ }^{11}$. These fibroblasts scaffold a fibrovascular provisional

\footnotetext{
${ }^{1}$ Department of Biomedical Engineering, University of Rochester, Rochester, NY, USA. ${ }^{2}$ Center for Musculoskeletal Research, University of Rochester Medical Center, Rochester, NY, USA. ${ }^{3}$ Department of Orthopaedics, University of Rochester Medical Center, 601 Elmwood Avenue, Box 665, Rochester, NY 14642, USA. ${ }^{4}$ UR Genomics Research Center (GRC), University of Rochester Medical Center, Rochester, NY, USA. ${ }^{5}$ Department of Biochemistry, University of Vermont, Burlington, VT, USA. ${ }^{6}$ These authors contributed equally: Jacob G. Kallenbach and Margaret A. T. Freeberg. ${ }^{\boxplus}$ email: hani_awad@urmc.rochester.edu
} 
matrix (granulation tissue) rich with collagen type III to partially restore tissue continuity. The remodeling phase, which is typically protracted, gradually replaces the provisional matrix with native tissue ECM components that are restructured to restore uninjured tissue mechanical properties, mediated by enzymatic activity of matrix metalloproteinases (MMPs). Time-dependent activation of Scx-expressing cells within the injury microenvironment is thought to constitute regenerative tendon healing in embryonic and neonatal tendons, whereas the activation of $\alpha-\mathrm{SMA}^{+}$myofibroblasts precipitates scar-mediated healing in adult tendon tissue ${ }^{12-15}$. Fibrotic scar persistence is thought to occur from an inadequate regenerative response from the native Scx-expressing cells, chronic presence of inflammatory cells and signals, heightened myofibroblast activity, and aberrant MMP remodeling ${ }^{16}$. Despite recent advances elucidating the cellular and molecular mechanisms of tendon healing, gaps in knowledge remain and have hindered the identification of novel therapeutic candidates that could prevent fibrosis and stimulate regenerative tendon healing.

Multifarious studies have identified the Murphy Roth's Large (MRL/MpJ) mouse as a promising mouse model of mammalian tissue regeneration due to its ability to restore native-like tissue structures and properties within the ear ${ }^{17}, \operatorname{skin}^{18}$, skeletal muscle ${ }^{19,20}$, articular cartilage ${ }^{13,21-23}$, and tendon ${ }^{24-26}$. Originally hypothesized that the immunomodulated systemic environment in MRL mice drove these regenerative phenotypes ${ }^{21}$, studies show the necessity of blood flow or vasculature to recapitulate native tissue structures such as skin ${ }^{27}$, while more avascular tissue injuries such as cartilage demonstrate minimal improvements ${ }^{21}$. Even though uninjured tendon is hypovascular, a recent patellar tendon defect study removed the central third of the patellar tendon from MRL and C57BL/6J (C57) mice and demonstrated that MRL patellar tendons recapitulate near native biomechanical properties ${ }^{25}$. More recent studies injured the patellar tendon with a $0.75 \mathrm{~mm}$ tendon mid-substance biopsy punch and again demonstrated that MRL patellar tendons heal to near native biomechanical properties with an attenuated inflammatory response, heightened MMP activity, and improved ECM alignment ${ }^{24,26}$. While the MRL enhanced regenerative potential within musculoskeletal tissues may depend on tissue-specificity and injury severity, recent transcriptomic ${ }^{28}$, proteomic ${ }^{29}$, and metabolomic ${ }^{20}$ techniques point to novel mechanistic insights. Altogether, the prevailing evidence suggests that the MRL tendon tissue healing response is improved, but the specific cellular and transcriptomic mechanisms of these biological effects remain to be elucidated.

In this study, we report histological and biomechanical data indicative of accelerated tendon repair in the MRL superhealer model in comparison to standard C57 mice. Our transcriptional analysis suggests that these biological effects are related to altered TGFB1 regulated gene sets negatively enriching cell cycle and senescence transcriptional pathways in the MRL tendons, and positively enriching inflammatory and ECM organization pathways in the C57 tendons.

\section{Results}

MRL tendons heal with reduced adhesions and improved biomechanical properties. We first sought to compare the extent of adhesion formation and repair strength of healing flexor tendons in the C57 and MRL mice. The healing of partially lacerated deep digital flexor tendons (DDFT) was qualitatively assessed using hematoxylin-stained axial tendon sections. In general, the MRL tendons appeared to respond to the injury with a reduced cellular response compared to the C57 tendons. Specifically, there were no gross morphological differences in the uninjured (Fig. 1A,E), where the tendon bundles were sparsely populated by cells, and the tendons were encased with a very thin synovial sheath (black arrows in Fig. 1a,e). Fourteen days post injury (dpi), injured tendons in both mouse strains experienced substantial hyperplasia in the surrounding synovial sheath (black arrows, Fig. 1b,f), while no significant changes in cell number in the tendon proper were generally observed (yellow arrows Fig. 1B,F). At $28 \mathrm{dpi}$, substantial increases in cell density in both the tendon bundle and the peritendinous adhesions were observed in the C57 mice but not the MRL mice (yellow arrows, Fig. 1C,G). At this stage, the synovial hyperplasia began to resolve in the MRL mice but persisted in the C57 mice (black arrows, Fig. 1c,g). By $56 \mathrm{dpi}$, the injured MRL tendons appeared to have restored uninjured morphology where the tendon bundle was sparsely cellular and encased by a thin synovial membrane (Fig. $1 \mathrm{H}, \mathrm{h}$ ). In contrast, while the cellularity of the C57 tendon bundle decreased, the surrounding synovial sheath still demonstrated a hyperplasia morphology at $56 \mathrm{dpi}$ (Fig. 1D,d). Quantification of the tendon perimeter adhered to the surrounding sheath and tissues (Fig. 1I), cellular density (Fig. 1J) in the tendon bundle demonstrated significant increases in adhesions and cellularity in the C57 tendons at $14 \mathrm{dpi}$ and $28 \mathrm{dpi}$, respectively, compared to the MRL tendons. In addition, the synovial space was significantly increased in the MRL tendon at 14 and 28 dpi compared to the C57 tendons (Fig. 1K), while the cross sectional area of the MRL tendons was significantly larger than the C57 tendon at all time points (Fig. 1L).

To test whether the changes in cell density over time and between strains are attributable to cell proliferation, immunohistochemical staining for Proliferating Cell Nuclear Antigen (PCNA) was performed (Fig. 2A) and demonstrated activation of cellular proliferation in the tendon bundle and the peritendinous tissues following injury, which peaked at $14 \mathrm{dpi}$. Furthermore, quantifying the percentage of $\mathrm{PCNA}^{+}$cells at all timepoints after injury revealed increased cellular proliferation in the healing C57 tendons relative to the MRL tendons (Fig. 2B).

The functional properties of injured tendons were evaluated using biomechanical testing, which involved displacement-controlled tensile stretching of the tendon to failure at $0.05 \mathrm{~mm} / \mathrm{s}$. In terms of the structural properties, the injury resulted in significant decreases in peak tensile force and stiffness at $14 \mathrm{dpi}$ in both the C57 and the MRL tendons. In contrast to the C57 tendons, which exhibited sustained reductions in peak tensile force and stiffness up to $56 \mathrm{dpi}$, the MRL tendons recovered uninjured levels of peak tensile force by $28 \mathrm{dpi}$ (Fig. 3A) and were significantly stiffer (Fig. $3 \mathrm{~B}$ ) than the C57 tendons at all time points up to $56 \mathrm{dpi}$. Since the crosssection area of the uninjured MRL tendons are approximately $20 \%$ larger than the C57 tendons, we computed the corresponding stress-strain tensile curves and determined the effects of injury on the size-independent material properties. At $14 \mathrm{dpi}$, the tensile strength (peak stress) of the MRL was significantly reduced compared 

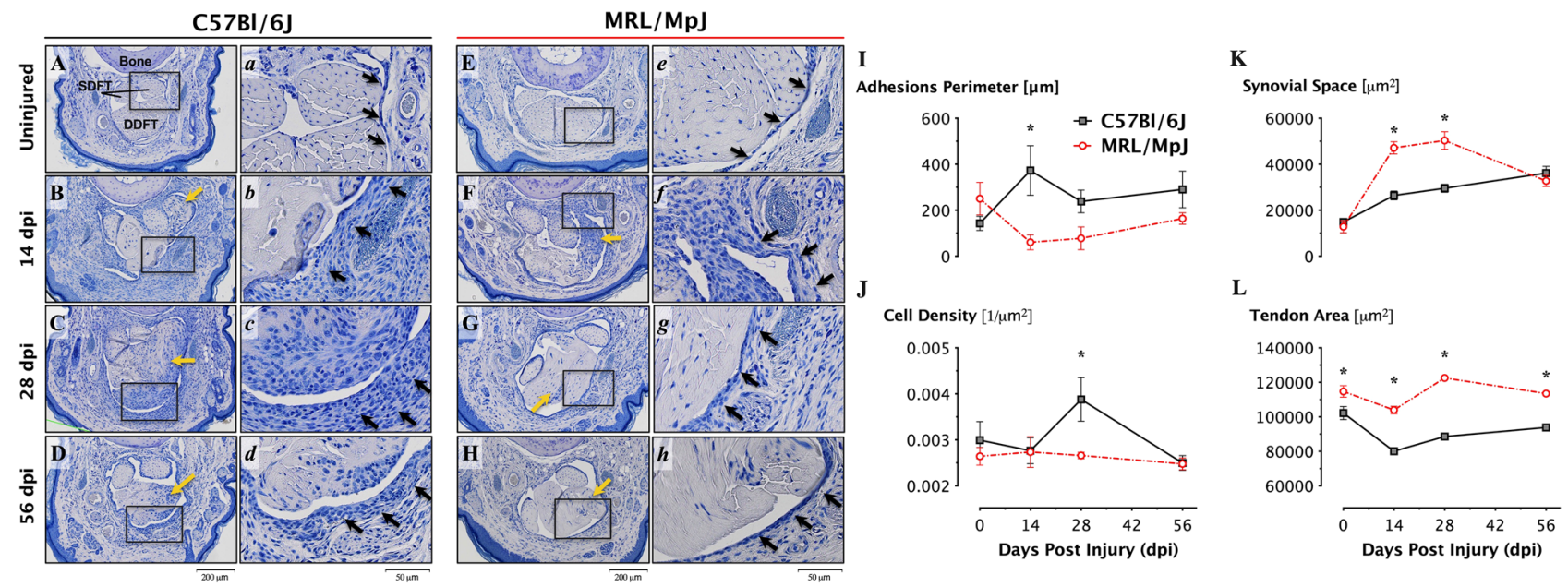

Figure 1. MRL flexor tendons heal with reduced peritendinous adhesions. Representative (2X) micrographs (A-H) of hematoxylin-stained cross-sections of the digits of C57 and MRL mice before and after zone II injury up to 56 days post injury (dpi). High magnification $(\times 20)$ regions of interest $(\mathbf{a}-\mathbf{h})$ are representative of five levels analyzed per tendon. Black arrows identify the thin synovial sheath in uninjured tendons, and the hyperplasia that ensues immediately after injury in both strains. The synovial sheath hyperplasia appears to persist in the C57 tendons up to $56 \mathrm{dpi}$, but resolves in MRL tendons by $28 \mathrm{dpi}$. Yellow arrows indicate regions of increased cellularity in the injured tendon and peritendinous tissues evident by the punctate, intense hematoxylin staining. Histomorphometric quantification of the healing tendon shows reduced adhesions (I) and tendon hyperplasia $(\mathbf{J})$, as well as increased synovial space $(\mathbf{K})$ and tendon area $(\mathbf{L})$ in the MRL mouse. The length of the tendon perimeter adhered to the subcutaneous tissue and cell density are significantly reduced at 14- and 28- dpi in the MRL, respectively ( $\mathrm{N}=4$ animals with $\mathrm{n}=3-5$ images (technical replicates) per animal).
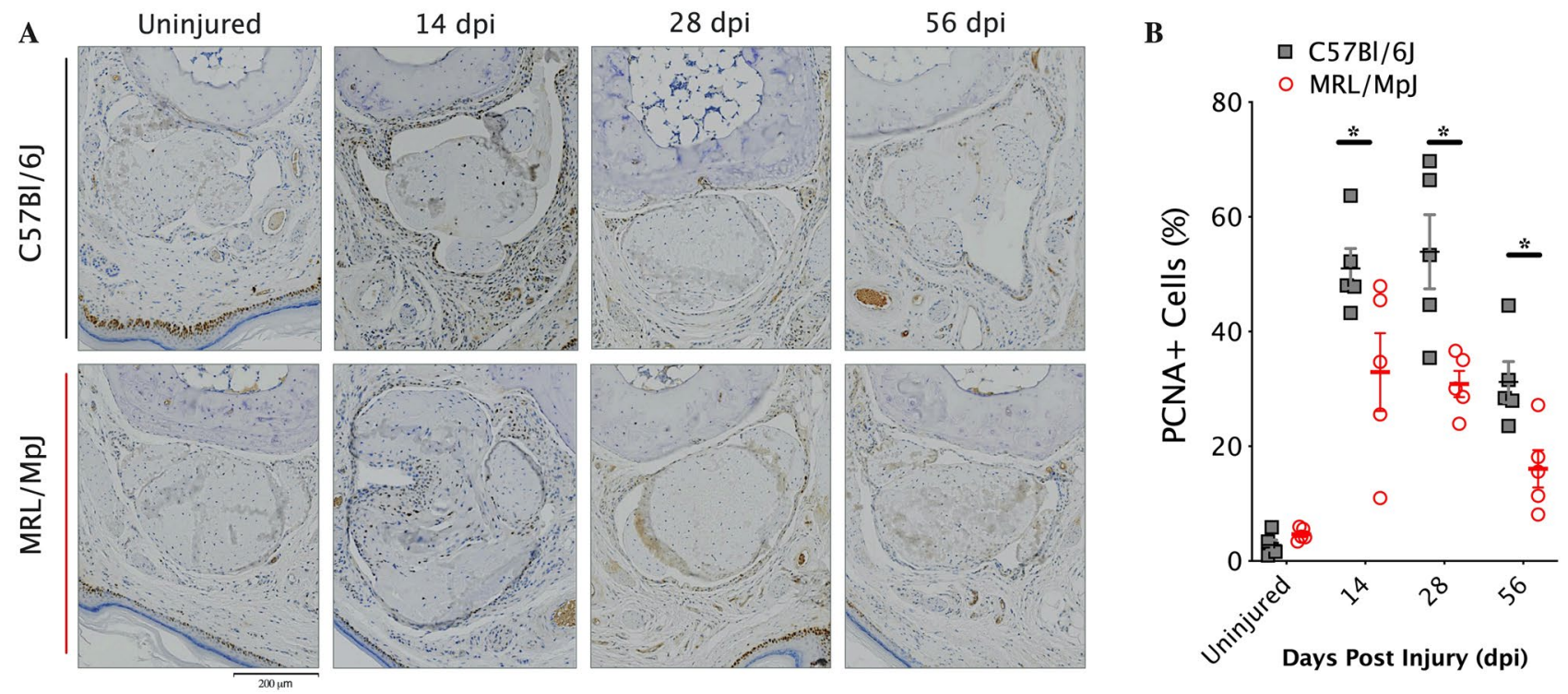

tive images of

Figure 2. MRL flexor tendons heal with reduced cellular proliferation. (A) Representative images of
proliferating cell nuclear antigen (PCNA) immunohistochemical (IHC) staining conducted before injury and over time post injury on $5 \mu \mathrm{m}$ transverse paraffin sections and counterstained with hematoxylin, and then quantified (B) for percent PCNA-positive cells within the tendon and the surrounding peritendinous tissues $(\mathrm{N}=5$ per group, with $\mathrm{n}=3-5$ images (technical replicates) per animal). Scale bar $=200$ microns.

to uninjured tendon and the C57 injured tendon. The tensile strength of the C57 continued to decline at 28 dpi but recovered at $56 \mathrm{dpi}$. In contrast, the MRL tendon recovered the tensile strength to levels comparable to uninjured tendon as early as $28 \mathrm{dpi}$ (Fig. 3C). Injury resulted in significant reductions in the elastic modulus of the C57 tendon, which did not improve over 56 dpi. While the elastic modulus of the uninjured MRL tendon was significantly reduced compared to the uninjured C57, injury resulted in reductions in the elastic modulus on 14,28 , and $56 \mathrm{dpi}$, which were not significantly different than uninjured tendon (Fig. 3D). Collectively, these 
A

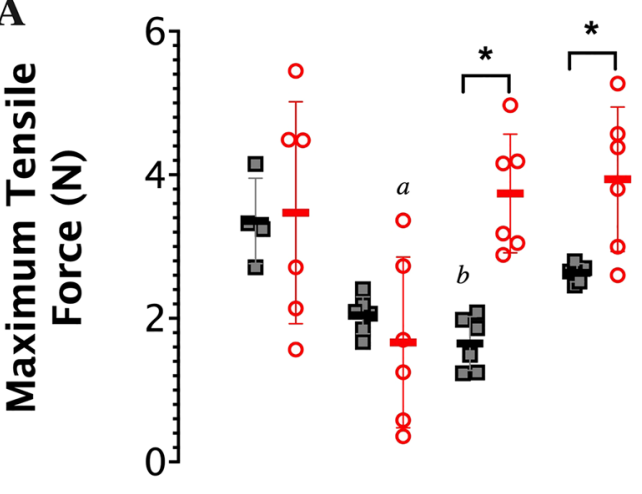

C

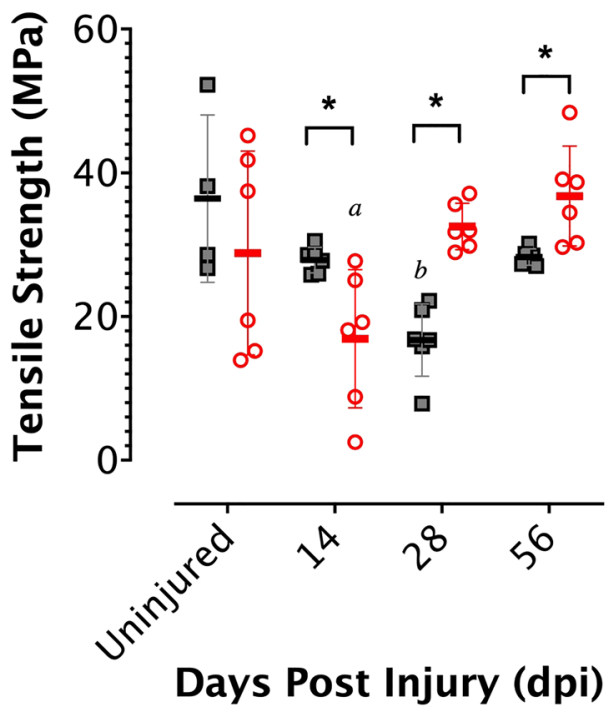

B

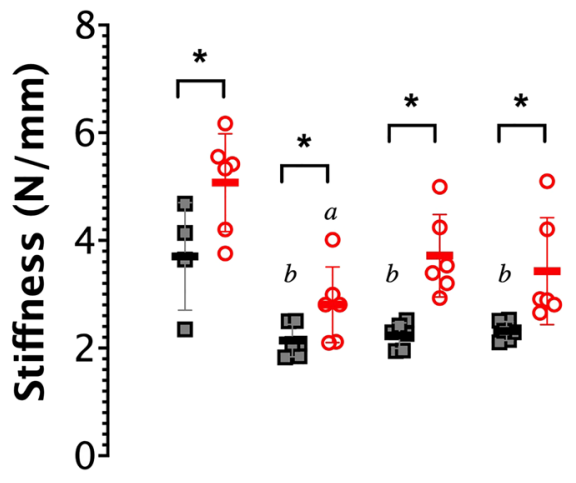

D

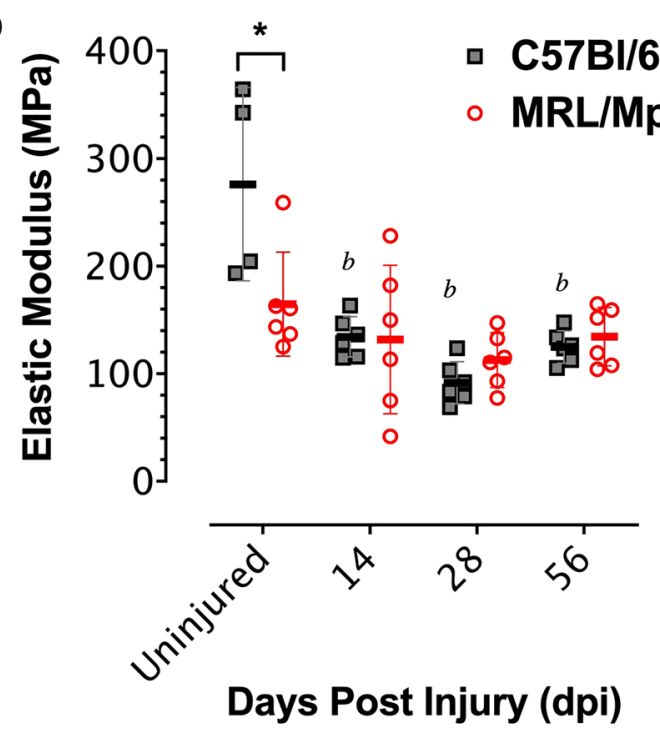

Figure 3. MRL flexor tendons heal with accelerated recovery of strength and stiffness (A) Maximum force, (B) strength, (C) stiffness and (D) elastic modulus determined from displacement-controlled tensile testing of uninjured and injured flexor tendons of $\mathrm{C} 57 \mathrm{BL} / 6 \mathrm{~J}$ and MRL mice $(\mathrm{N}=4-6)$. Asterisks indicate significant mouse strain differences $(p<0.05)$. a and b indicate significant differences $(p<0.05)$ from uninjured within C57 and MRL mouse strains respectively.

data support the conclusion that MRL flexor tendons heal with reduced peritendinous adhesions and cellular proliferation and accelerated recovery of strength and stiffness.

Altered inflammatory, fibrotic, and cell cycle regulation in C57 and MRL tendon healing. To gain insights into the differences in the biological drivers of the tendon injury response between the C57 and MRL mice, we performed next generation sequencing of bulk tissue RNA (RNA-seq) at 7 days post-injury, which we reasoned to be a time point that defines the transition from the acute inflammatory phase to the reparative phases. Based on a twofold change $( \pm 2 \mathrm{FC})$ and adjusted $p$ value $<0.05$ differential expression criteria relative to healthy uninjured tendon, 891 genes were differentially expressed (594 upregulated and 297 downregulated) in the C57 tendons, and 1358 genes were differentially expressed (747 upregulated and 611 downregulated) in the MRL tendons. Of those, 406 genes (DEG) were differentially expressed, relative to respective uninjured controls, in both mice. We identified 22 significantly enriched Reactome pathways in the injured C57 tendon and 51 significantly enriched pathways in the injured MRL tendon based on False Discovery Rate (FDR) $<0.05$. Functional annotation of these enriched gene sets identified remarkable differences in the transcriptional activity, wherein the gene sets enriched in the C57 injured tendon were functionally linked primarily to fibrosis, immune system signaling and extracellular matrix (ECM) organization (Fig. 4A), while the gene sets enriched in the MRL injured tendon were dominated by cell cycle pathways (Fig. 4B). Closer inspection of the top 15 Reactome pathways enriched in the C57 injured tendons (Fig. 5A) identified cytokine signaling, including interleukins 4,10 , and 13, and neutrophil degranulation as significantly enriched immune system pathways, and collagen formation (biosynthesis and assembly), ECM proteoglycans, ECM degradation, and integrin and non-integrin cell-ECM interactions as significantly enriched ECM organization pathways. In contrast, the top 15 Reactome pathways enriched in the MRL injured tendons (Fig. 5B) identified numerous cell cycle pathways related to mitotic phase, cell cycle checkpoints, and G1-S phase transition, as well as cell signaling by Rho GTPases and immune system signaling by interleukins and neutrophil degranulation. 
A

\section{C57BI/6J}

0.20

0.15

$\frac{0}{0}$

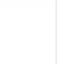

0.10

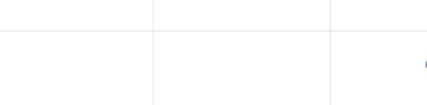

$\circ$

Reactome Pathway Root

Cell Response to External Stimuli Extracellular Matrix Organization Immune System

-Log(FDR)

2

4

6

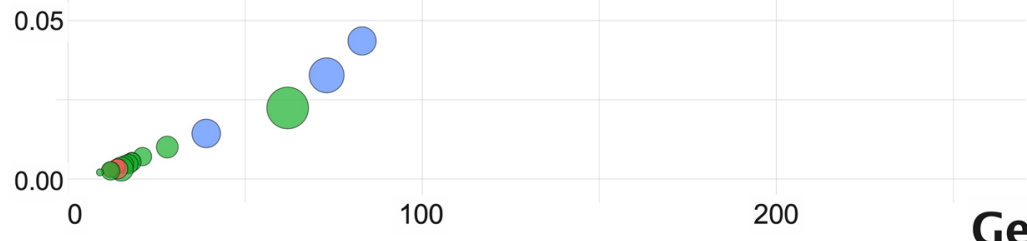

B

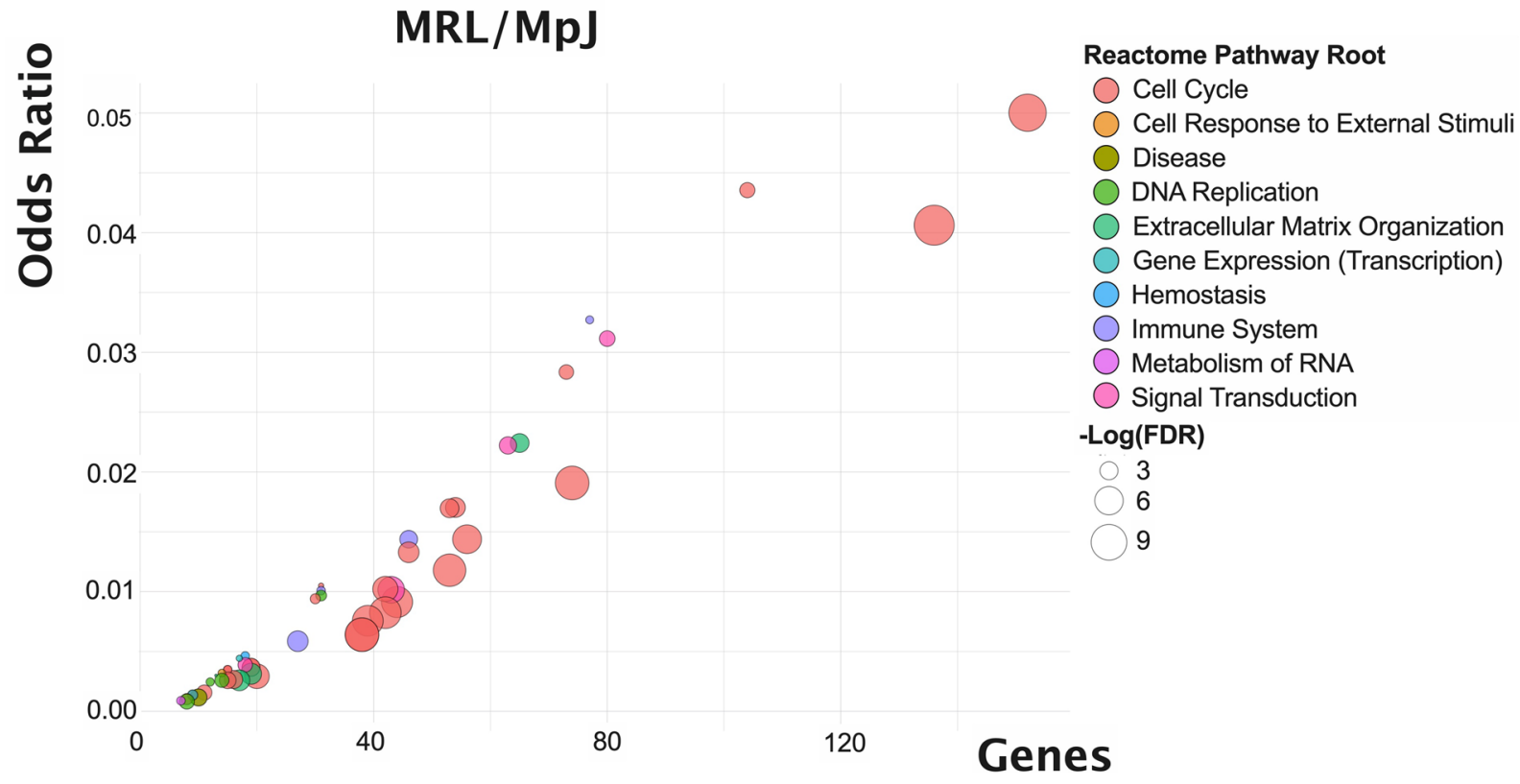

Figure 4. Functional annotation based on Reactome Pathways enrichment of 891 and 1358 differentially expressed genes (DEG) at 7 days post tendon injury in the C57 and MRL mice, respectively. Bubble plots of number of gene terms ( $\mathrm{x}$-axis) versus odds ratio (y-axis) represent 22 significantly enriched pathways in the injured C57 tendon (A) and 51 significantly enriched pathways in the injured MRL tendon (B) based on False Discovery Rate (FDR) $<0.05$.

To investigate differences in activation of myofibroblasts and macrophages as drivers of fibrotic scarring that could explain these transcriptional differences, histology and immunofluorescent staining against $\alpha$-SMA+ myofibroblasts, F4/80+ macrophages, and BCL-2 expressing cells were performed on C57 and MRL tendons at 7 dpi. Visual examination of Masson's trichrome stained injured tendon sections at this time point did not reveal any significant morphological differences in the injured tendons (Fig. 6A,B), except for increases in the vascularization of the scar tissue immediately surrounding the injured C57 tendon, which was notable in $\alpha-S M A+$ smooth muscle cells surrounding the blood vessels (Fig. 6C). In addition to the increased vascularization in the C57 scars, activated ( $\alpha$-SMA+) myofibroblasts and F4/80+ macrophages could be observed in both the injured C57 tendon and the surrounding scar tissue (Fig. 6C and insets). Of note was the observation of a subset of cells coexpressing both SMA and F4/80 in the injured C57 tendon (yellow staining in Fig. $6 c^{\prime}$ ), which could implicate the activation of macrophage-myofibroblast transition (MMT) as a feature of fibrotic tendon healing. In contrast, 


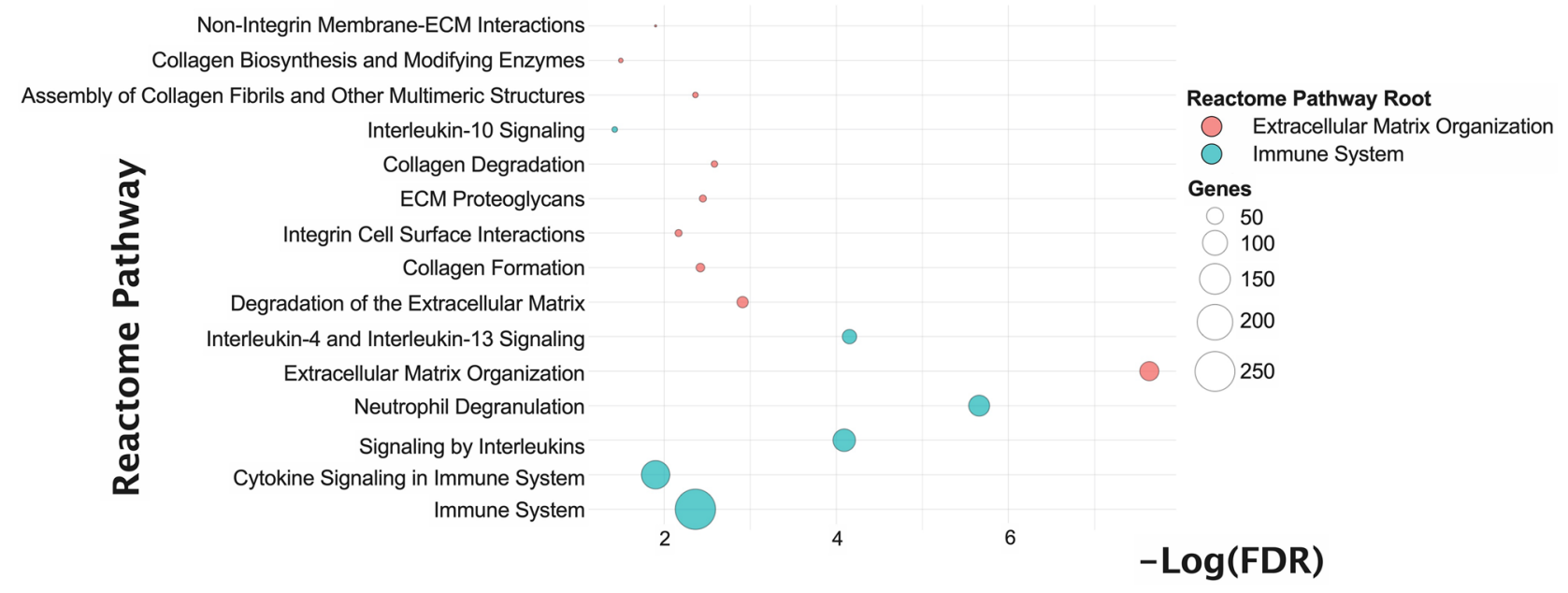

B

\section{$\mathrm{MRL} / \mathrm{MpJ}$}

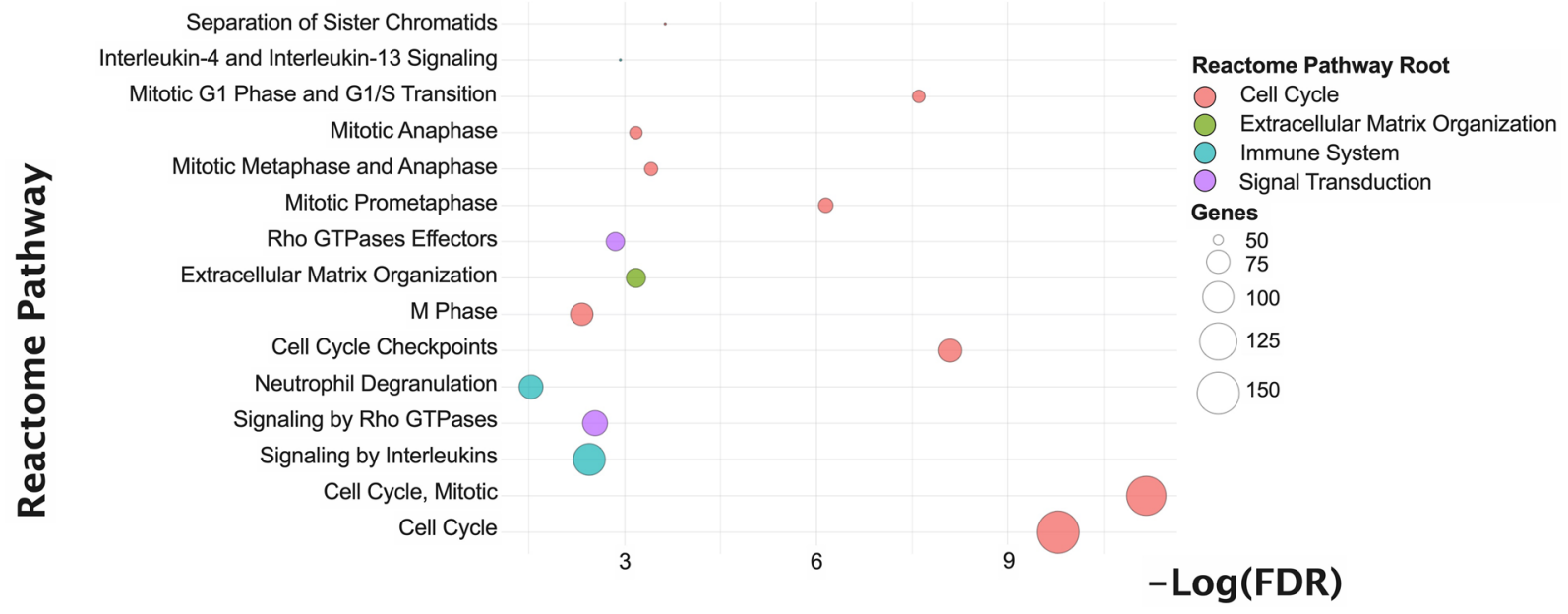

Figure 5. Bubble plots of the top 15 Reactome pathways enriched in the injured C57 tendon (A) and the MRL tendon (B), respectively. Bubble fill color represents the Reactome Pathway Root, while the size of the bubble represents $-\log _{10}(F D R)$ in $A \& B$ and the number of genes represented in the enriched pathway in $C \& D$, respectively.

fewer $\alpha$-SMA+ myofibroblasts and F4/80+ macrophages could be observed in the injured MRL tendon, but were abundant in the surrounding scar tissue (Fig. 6D and insets). No localized expression of $a$-SMA and F4/80 could be observed in the injured MRL tendon. BCL-2 expression was notably abundant in the injured C57 tendon, but appeared to be reduced in the surrounding scar tissue (Fig. 6E and insets) as well as in the MRL injured tendon and surrounding scar tissues (Fig. 6F and insets).

Dichotomous roles for TGB1 in the C57 and MRL tendons. Ingenuity pathways analysis (IPA) of active or inhibited upstream transcriptional regulators were also examined to provide additional insights into the observed gene expression changes driving the altered mechanobiology of tendon healing. In the C57 injured tendons, the top 20 activated and inhibited upstream regulators (Fig. 7A) were associated with 184 and 62 unique genes, respectively, that enriched Gene Ontology (GO) terms related to chemotaxis and cell motility and migration, ECM organization, sprouting angiogenesis and blood vessel morphogenesis, positive regulation of cell growth and proliferation, inflammatory responses, collagen catabolism and regulation of phosphatidylinositol- and ERK1/2-signaling (Table S1). In the MRL injured tendon, the top 20 activated and inhibited upstream regulators (Fig. 7B) were associated with 325 and 304 unique genes, respectively, that enriched GO terms related to regulation of cell cycle, including mitotic cell cycle, sister chromatid cohesion, G1/S transition of mitotic cell cycle, mitotic cytokinesis, DNA replication, chromosome segregation, regulation of cell cycle, microtubulebased movement, metaphase plate congression, mitotic spindle organization, G2/M transition of mitotic cell 

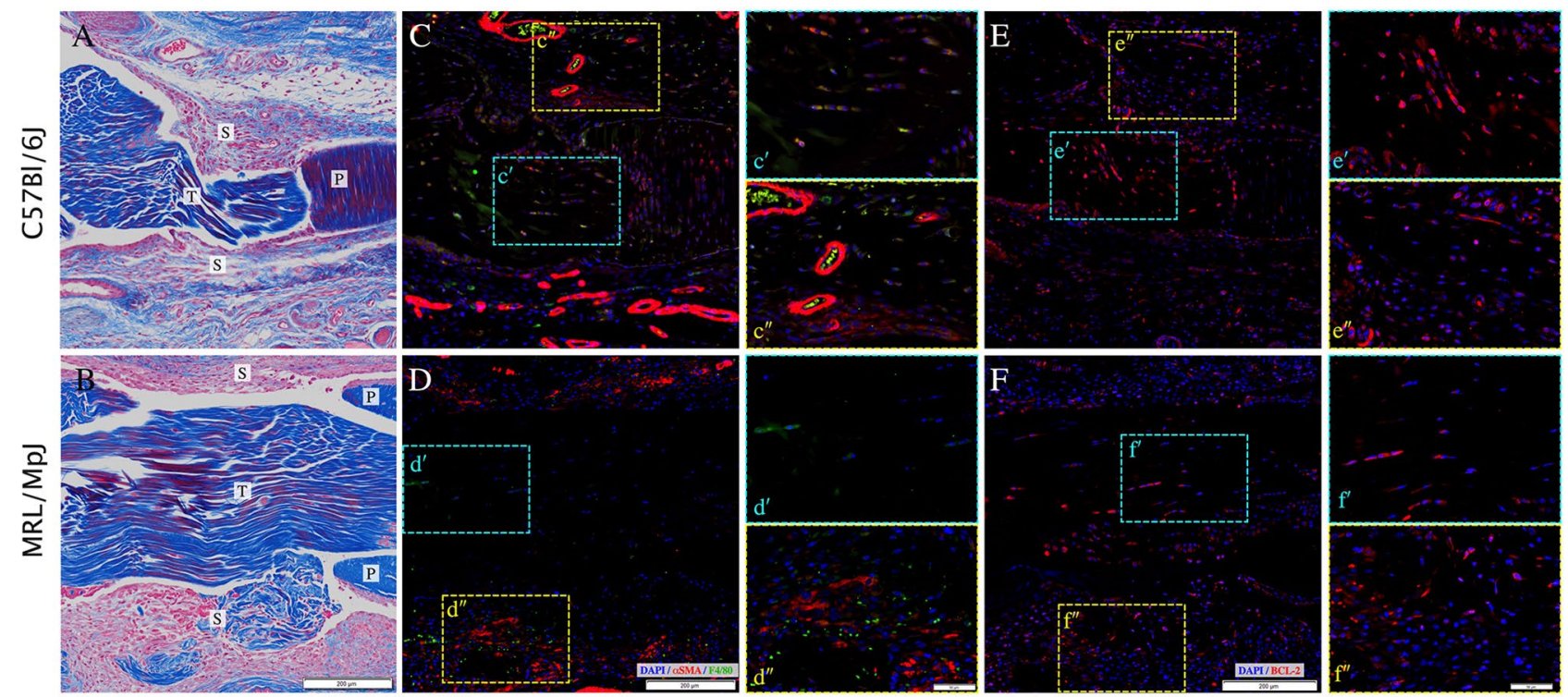

Figure 6. MRL Tendons heal with reduced myofibroblast and macrophage activation and reduced BCL2 expression at 7 dpi. Histology using Masson's trichrome $(\mathbf{A}, \mathbf{B})$ and immunofluorescent (IF) staining against a-SMA+ myofibroblasts and F4/80+ macrophages $(\mathbf{C}, \mathbf{D})$, and BCL-2 expressing cells (E,F) were performed on injured C57 and MRL tendons at $7 \mathrm{dpi}$. IF shows increased vascularization in the C57 scars and activated (a-SMA+) myofibroblasts and F4/80+ macrophages in both the injured C57 tendon and the surrounding scar tissue (Insets $c^{\prime}$ and $c^{\prime \prime}$ ). A subset of cells co-expressing both SMA and F4/80 in the injured C57 tendon (Inset $\left.c^{\prime}\right)$. Fewer $\alpha$-SMA+ myofibroblasts and F4/80+ macrophages are observed but no localized expression of $\alpha$-SMA and F4/80 is evident in the injured MRL tendon (Inset $\mathrm{d}^{\prime}$ ), but SMA + myofibroblasts and F4/80+ macrophages were abundant in the surrounding scar tissue (Inset $\mathrm{d}^{\prime \prime}$ ). BCL-2 expression was notably abundant in the injured C57 tendon (Inset $\mathrm{e}^{\prime}$ ), but appeared to be reduced in the surrounding scar tissue (Inset $\mathrm{e}^{\prime \prime}$ ) as well as in the MRL injured tendon and surrounding scar tissues (Insets $\mathrm{f}^{\prime}$ and $\mathrm{f}^{\prime \prime}$ ). Scale bars $=200$ micron in main figures and 50 microns in insets. In panels (A) and (B), T denotes tendon, $\mathrm{P}$ denotes pulley, $\mathrm{S}$ denotes scar.

A

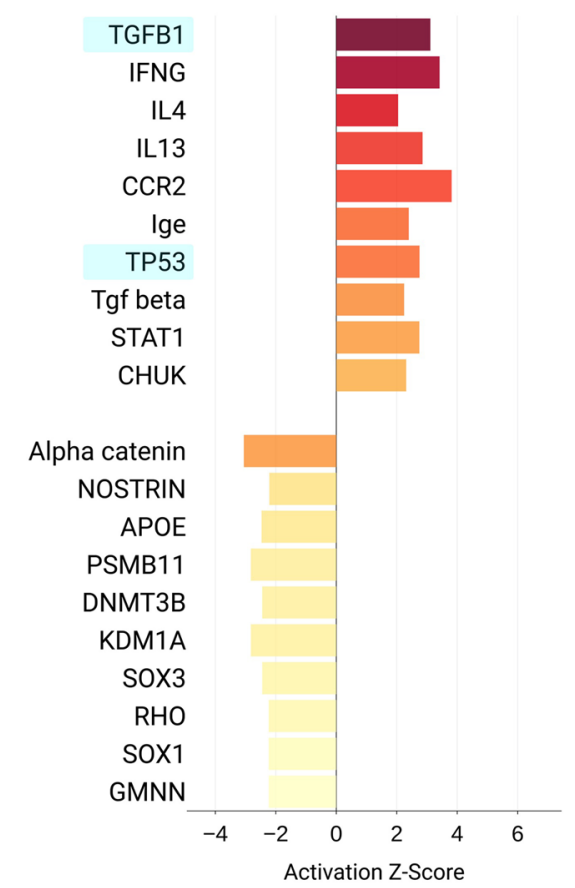

B

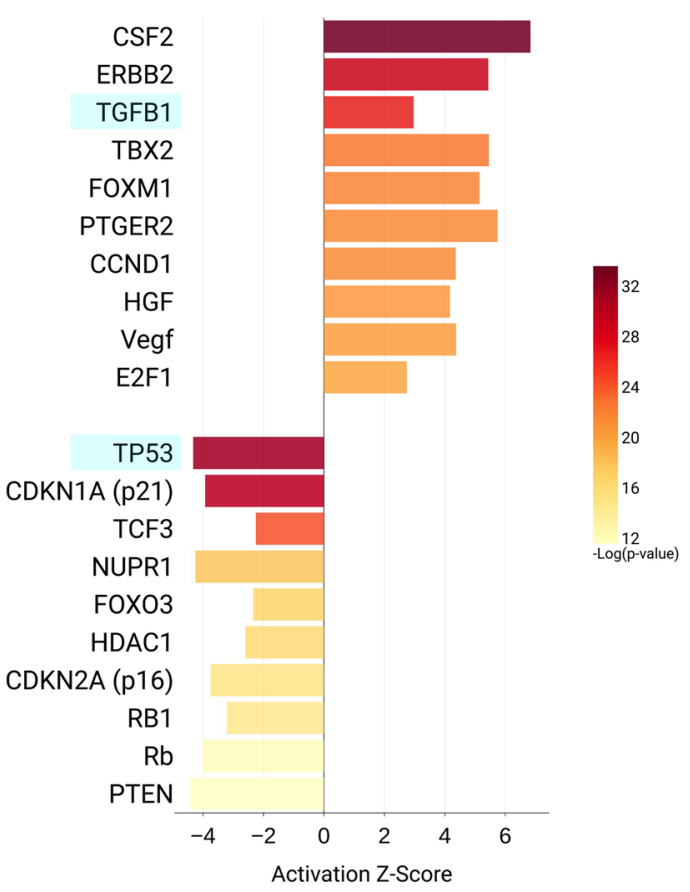

Figure 7. Ingenuity Pathway Analysis (IPA) Upstream Regulators during tendon healing in the C57 (A) and MRL (B) mice. Activation (Z-score $\geq 2$, $p$ value of overlap $<0.05$ ) or inhibition $(Z$-score $\leq-2, p$ value of overlap $<0.05)$ was determined relative to uninjured tendon gene expression in either strain. 
cycle, negative regulation of cell cycle, and extracellular matrix organization (Table S2). Of particular note, was the inhibition of upstream regulators TP53, CDKN1A (p21), CDKN2A (p16), which are associated with DNA damage response and cell cycle arrest.

TGFB1 was a significantly activated Upstream Regulator in both the C57 (Activation Z-score $=3.11$ and $p$ value $=6.4 \times 10^{-13}$ ) and MRL (Activation $Z$-score $=2.97$ and $p$ value $=1.1 \times 10^{-27}$ ) injured tendons. GO functional annotation revealed that activation of TGF- $\beta 1$ drives different biological pathways in the C57 and MRL injured tendons. In the C57 injured tendons, the 76 target genes of TGFB1 (Table S3) enriched GO pathways related to cytokine and chemokine signaling, ECM organization, and cell motility and migration (Fig. 8A). In contrast, 160 target genes of TGFB1 in the MRL (Table S4) enriched GO pathways associated with not only ECM organization and cytokine signaling but mostly with regulation of cell cycle, mitosis, and DNA binding and transcription (Fig. 8B).

Reduced circulating cytokines and chemokines in the MRL injured mice. We have previously shown that the putative senescence marker plasminogen activator inhibitor 1 (PAI-1), downstream of TGF- $\beta$, is elevated in fibrotic tendon tissue in humans and mice ${ }^{30}$. In subsequent studies, we observed increases in the prosurvival signal BCL-2 in injured tendon, which appeared to be linked to inflammatory nuclear factor $\kappa \mathrm{B}$ (NF$\kappa \mathrm{B})$ signaling ${ }^{31}$. Therefore, in order to evaluate the roles of the immune response and systemic inflammatory signaling on the tendon healing, sera were extracted from whole blood draws from mice at different time points up to $14 \mathrm{dpi}$ and analyzed for senescence associated secretory phenotype (SASP) proteins including inflammatory cytokines/chemokines, TGF- $\beta$ s, and MMPs. In general, C57 and MRL mice exhibit similar circulatory concentrations of serum proteins in their uninjured state (Fig. 9A). Upon injury, the temporal changes in the circulating proteins in the $\mathrm{C} 57$ mice were in general more pronounced than in the MRL mice. Analyzed proteins were clustered in 4 major classes based on Pearson's correlations (Fig. 9B). In the first cluster, the chemokine CXCL9 (MIG) showed no significant difference over time in the C57 but increased significantly over time in the MRL injured tendons. Cluster 2 proteins showed no significant differences over time or between C57 and MRL mice, which included TGF- $\beta$ s $(1,2,3)$, MMP-3 and CCL 4 (MIP-1 $\beta$ ). Cluster 3 proteins, which included IL-1 $\alpha$, -12 (P40), -13, MMP-2 and -8, CSF1 (M-CSF) and 3, CCL5 (RANTES) and 11 (Eotaxin), CXCL2 (MIP-2) and 5 (LIX), were significantly higher in the C57 mice compared to the MRL mice, but did not vary significantly over time. Cluster 4 proteins showed significant changes over time in the C57 mice only, peaking at either $1 \mathrm{dpi}$ (IL-2 and -9, MMP-12, and CCL3 (MIP-1 $\alpha$ )) or 5 dpi (IL-1 $\beta,-2,-4,-5,-6,-7,10,-12$ (P70), -17, TNF- $\alpha$, LIF, MMP-9, CCL2 (MCP-1) and CXCL1(KC) and 10 (IP-10)). In contrast circulation levels of these proteins were significantly lower and remained mostly unchanged over time in the MRL mice. Protein association networks of cluster 3 (Fig. 9C) and cluster 4 (Fig. 9D) circulating proteins visualized in String DB identified strong functional associations, which involved cytokine signaling in immune system and more specifically signaling by interleukins, chemokine signaling and activation of matrix metalloproteinases.

\section{Discussion}

Development of fibrotic flexor tendon adhesions, particularly in zone II of the hand ${ }^{32}$, limits their natural gliding function, strength, and intricate joint flexion ${ }^{33,34}$. Recent biological advances in scarless repair of tendon injuries ${ }^{24,26,35}$ have begun to elucidate the complex cellular and transcriptional interplay in restoring native tissue properties to injured tendons. For example, previous studies demonstrate that MRL tendon injuries heal to near normal biomechanical properties ${ }^{25}$, have reduced infiltrating immune cells ${ }^{25}$, attenuated inflammatory expression $^{24,36}$, lower catabolic enzyme activity ${ }^{28}$, heightened MMP activity ${ }^{24}$, and improved ECM alignment ${ }^{26}$. These reports are consistent with our current findings in flexor tendons, but the biological drivers of this regenerative tendon healing phenotype have hitherto not been fully understood.

The objective of our study was to uncover the mechanisms of improved healing of MRL flexor tendon injuries to understand better the limitations of natural healing in common C57 mouse tendons. We first investigated the longitudinal histomorphometry and biomechanical properties of MRL and C57 mice flexor tendons after a zone II partial laceration injury. We demonstrated that the MRL flexor tendons heal with reduced tenocyte cellularity, reduced peritendinous adhesions, and accelerated recovery of their biomechanical properties of tensile strength, stiffness, and elastic moduli. We then performed bulk tissue transcriptomic analysis using next-generation RNA sequencing (RNA-Seq) to characterize enriched transcriptional pathways in the early reparative phase. These experiments uncovered dichotomous roles for TGFB1 in the healing of C57 and MRL tendons. Specifically, we identified TGFB1 as a major driver of positive enrichment of cytokine signaling, neutrophil degranulation, and ECM degradation pathways in injured C57 flexor tendons, and, by contrast, negative enrichment of cell cycle regulation and senescence pathways in the injured MRL flexor tendons. To validate these transcriptional observations, we investigated the systemic inflammatory response by analyzing the cytokines, chemokines, and growth factors in sera extracted from peripheral blood at different phases of healing. These assays revealed significantly lower circulatory concentrations of SASP proteins in the MRL relative to the C57 despite comparable baseline (day 0 ) serum concentrations. These findings provide insights into novel molecular pathways in tendon healing that could be therapeutic targets for regenerative tendon repair.

TGF- $\beta$ is critical to the modulation of tendon healing and the effects of its isoforms are quite distinct. In flexor tendons, TGF- $\beta 1$ expression is auto-inductive and is initially produced by inflammatory cells ${ }^{37}$. TGF- $\beta 1$ stimulates fibroblast proliferation and migration, synthesis of collagen and fibronectin, inactivation of MMPs ${ }^{38}$ and myofibroblast differentiation ${ }^{12}$. Chang et al. reported increased expression of TGF- $\beta 1$ in both tenocytes and infiltrating fibroblasts and inflammatory cells from the tendon sheath in a rabbit zone II flexor tendon healing model $^{39}$. The inflammatory cells stimulate synovial and epitenon fibroblasts, partly through TGF- $\beta 1$, to produce fibronectin, a marker of scarring ${ }^{37}$. The role of TGF- $\beta 1$ in scar formation is accentuated by the observation that 
positive regulation of epithelial to mesenchymal transition (GO:0010718) extracellular matrix disassembly (G0:0022617) endothelial cell migration (G0:0043542)

positive regulation of peptidyl-tyrosine phosphorylation (G0:0050731) transmembrane receptor protein tyrosine kinase signaling pathway (G0:0007169) positive regulation of epithelial cell proliferation (GO:0050679) regulation of chemokine secretion (G0:0090196) regulation of bone resorption (G0:0045124) myeloid leukocyte differentiation (GO:0002573) positive regulation of epithelial cell migration (GO:0010634) epithelial cell migration (GO:0010631) branching involved in blood vessel morphogenesis (GO:0001569) regulation of bone remodeling (GO:0046850) positive regulation of multicellular organismal process (GO:0051240) regulation of cell migration (GO:0030334) cellular response to cytokine stimulus (GO:0071345) positive regulation of cell motility (GO:2000147) positive regulation of cell migration(G0:0030335) extracellular matrix organization (G0:0030198) cytokine-mediated signaling pathway (G0:0019221)

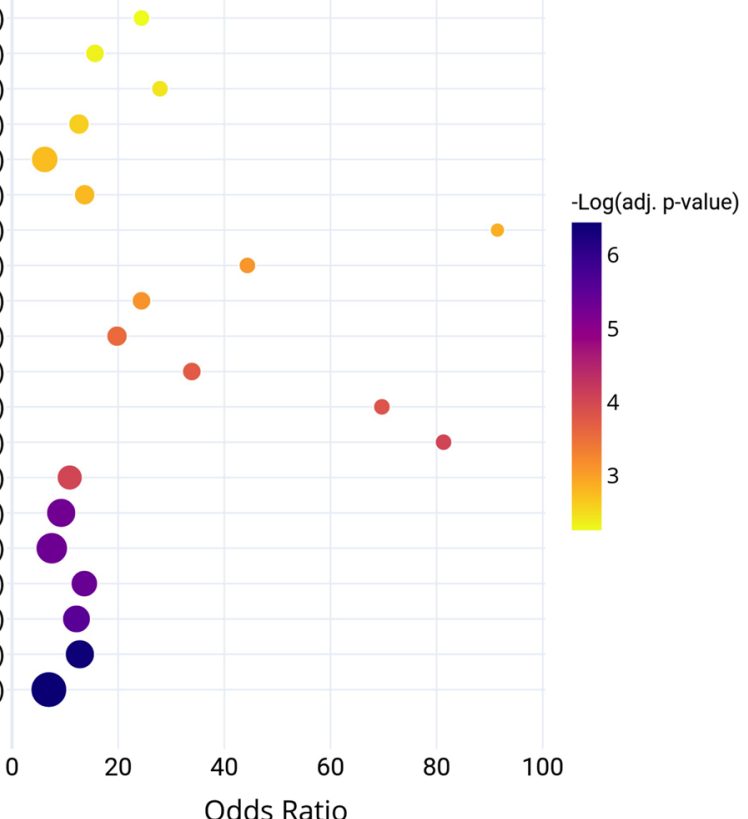

MRL/MpJ

positive regulation of cell proliferation (G0:0008284) positive regulation of DNA replication (GO:0045740) mitotic sister chromatid segregation (GO:0000070) positive regulation of gene expression (G0:0010628) negative regulation of cellular macromolecule biosynthetic process (GO:2000113) positive regulation of transcription, DNA-templated (G0:0045893) regulation of transcription, DNA-templated (GO:0006355) regulation of cyclin-dependent protein serine/threonine kinase activity (GO:0000079) negative regulation of cell proliferation (GO:0008285) regulation of cell proliferation (GO:0042127) mitotic cell cycle phase transition (G0:0044772) kinetochore organization (G0:0051383) negative regulation of cellular process (G0:0048523) cytokine-mediated signaling pathway (GO:0019221) regulation of DNA binding (GO:0051101) regulation of mitotic cell cycle (GO:0007346) regulation of epithelial cell proliferation (G0:0050678) extracellular matrix organization (G0:0030198) negative regulation of cell cycle (GO:0045786) regulation of cell cycle (GO:0051726)

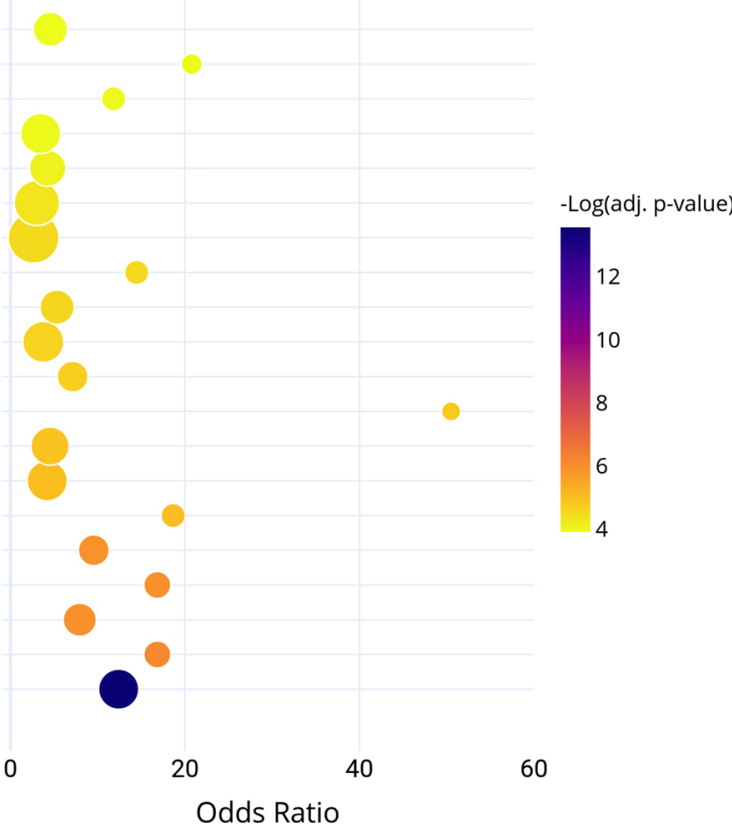

Figure 8. Biological Process Gene Ontology (GO) of 76 genes contributing to the activation of TGFB1 Upstream Regulator in the healing C57 tendon (A). Biological Process Gene Ontology (GO) of 160 genes contributing to the activation of TGFB1 Upstream Regulator in the healing MRL tendon (B). Bubble area corresponds to the number of genes in the enriched GO biological process.

while fetal cutaneous wound healing and tendon repair are scar-free $e^{40,41}$, the in utero administration of TGF- $\beta 1$ to fetal rabbit wounds results in fibrotic scarring similar to that observed in adult rabbits ${ }^{41}$. In our study, we did not observe changes in circulating TGF- $\beta 1$ levels over time or between the C57 and MRL mice. However, we 
A

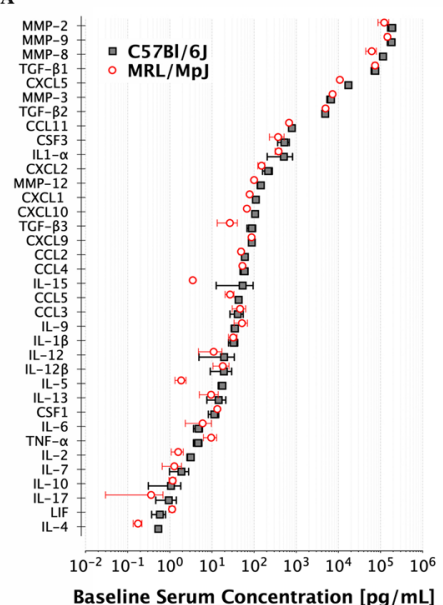

B

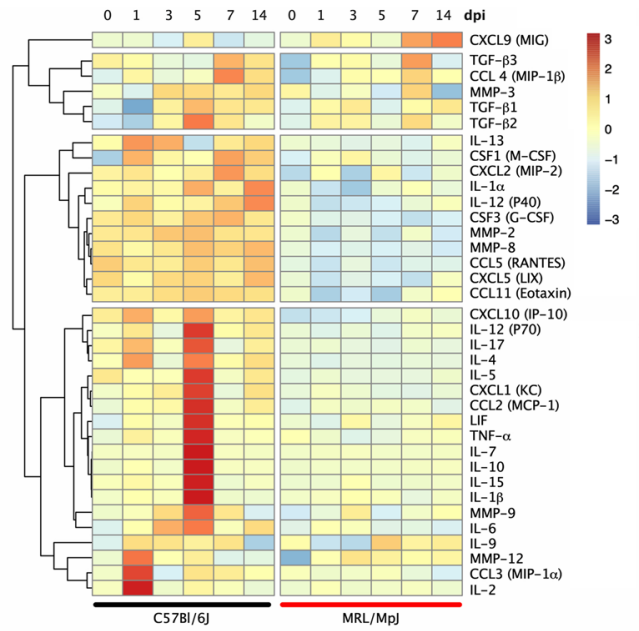

C

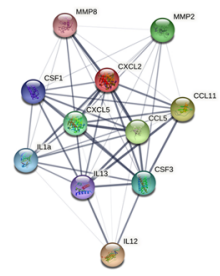

D

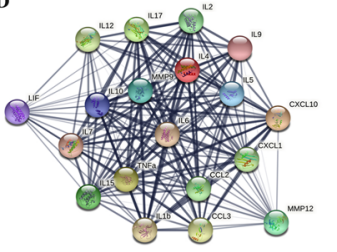

Edge Confidence $\bigcirc$ low (0.15) 0 medium (0.40) high (0.70)

Figure 9. Serum protein analysis indicates temporal systemic upregulation of several circulating enzymes, cytokines, and chemokines in the C57 mice following tendon injury, but not in the MRL mice. (A) Baseline concentrations of serum proteins pre-injury plotted in order of concentration shows no significant differences between C57 and MRL mice. (B) Heatmaps for circulating serum enzymes, cytokines, and chemokines concentration (normalized to day 0 C57 controls) changes over time post flexor tendon injury (dark red indicates $>+$ threefold increase and dark blue indicated $>-$ threefold decrease compared to preinjury serum concentration). Dendrogram represent clustering based on Pearson's correlations. Each box represents the average of $\mathrm{N}=3$ mice at each time point. String DB representation of physical and functional association network of cluster 3 (C) and cluster 4 (D) proteins.

did observe dichotomous downstream effects of TGFB1 gene regulatory networks between the C57 and MRL healing tendons locally. In particular, genes regulated by TGFB1 in the C57 tendons positively enriched pathways relevant to cytokine signaling in chemotaxis and inflammation, ECM organization, angiogenesis, and epithelial to mesenchymal transition, whereas the TGFB1 regulated genes in the MRL tendons negatively enriched pathways predominately related to cell cycle regulation and senescence. These results corroborate reports of dual roles for TGF- $\beta$ in different contexts, such as being protective and cytostatic against tumorigenesis but also a promoter of tumor progression, invasion, and metastasis in established tumors ${ }^{42}$. Dual roles of TGF- $\beta$ have also been reported in autoimmune-induced tissue damage, wherein reduced TGF- $\beta$ signaling in immune cells can lead to inflammatory dysregulation triggering the production of TGF- $\beta$ in target organs, which subsequently leads to fibrogenesis and organ failure ${ }^{43}$. It is possible that the effects of TGF- $\beta$ in the injured tendon are intertwined with the inflammatory cytokines and chemokines in the site of injury and systemically. Particularly intriguing was the detection of cells positive for both F4/80 and a-SMA in the C57 injured tendons but not the MRL tendons. This observation could be indicative of macrophage-myofibroblast transition (MMT), which is implicated as a mechanism of chronic inflammation and progressive fibrosis in kidney ${ }^{44}$ but never reported in tendon repair. Previous studies have demonstrated the role of TGF- $\beta /$ Smad 3 signaling in regulating the transition of bone marrow-derived macrophages into myofibroblasts during tissue fibrosis ${ }^{45}$. It is also possible that intrinsic genetic differences in the tendon fibroblasts and infiltrating inflammatory cells could explain the dichotomous responses downstream of TGFB1. A third possibility is that the innate tissue properties in the MRL mouse tendon ${ }^{26} \mathrm{can}^{-}$ modulate the effects of TGF- $\beta 1$ resulting in accelerated repair. These possibilities warrant further investigation in future studies.

RNA-Seq analyses revealed negative enrichment of cell cycle regulation in the MRL tendons compared to the C57 healing flexor tendons who exhibited positive enrichment of inflammatory and ECM organization pathways, driven differentially by TGFB1 regulated gene sets. The increased responsiveness of injured C57 tendons to circulating inflammatory cytokines, chemokines, and growth factors may be associated with its positive enrichment of cell cycle evidenced by cyclin D1 (ccnd1) pathways. In contrast, injured MRL tendons negatively enriched pathways associated with cell cycle regulators TP53, CDKN1A (p21), CDKN2A (p16), RB1, FOXO3, and PTEN. The enrichment of these cell cycle regulators is associated with cellular senescence ${ }^{46}$. Cell cycle regulation and cellular senescence have recently become therapeutic targets in a number of conditions including fibrosis. Specifically, the cell cycle regulator p53 is induced in multiple fibrotic conditions such as idiopathic pulmonary fibrosis (IPF) and kidney fibrosis. In the absence of p53, bleomycin induced pulmonary fibrosis is prevented ${ }^{47}$ and its absence reduces chronic renal injury ${ }^{48}$. Furthermore, the antifibrotic agent used to treat IPF, Pirfenidone, has been shown to induce cell cycle arrest ${ }^{49,50}$. Reduced cell apoptosis, cell cycle regulation through p21 ( $c d k n 1 a)^{51}, \mathrm{p} 53(\operatorname{trp53})$, and p16 ( $\left.c d k n 2 a\right)$, increased cell proliferation ${ }^{52}$, and enhanced stem cell function ${ }^{53}$ may command augmented regenerative capacity.

While the prolonged systemic response in the C57 is surprising given the focal nature of the injury, the diminished response in the MRL is likely associated with decreased recruitment of inflammatory cells to the injury site similar to the scar-free healing of fetal wounds ${ }^{54}$. Interestingly, chemokine (C-X-C motif) ligand 9, CXCL9 is the single cytokine in cluster 1 and is transiently upregulated in MRL healing. In other fibrotic conditions increased CXCL9 has been beneficial to patient function and reduced fibrosis ${ }^{55,56}$. A study of serum cytokine 
levels of patients with hypersensitivity pneumonitis pulmonary fibrosis demonstrated a worsening lung function with reduced levels of CXCL9 ${ }^{55}$. Additionally, systemic administration of CXCL9 prevented the development of CCL4 induced liver fibrosis in a murine model $^{56}$. Altogether, the observed elevation of CXCL9 in the MRL sera after injury points to a potential therapeutic benefit of CXCL9 administration, which merits testing in tendon injury. In addition, proteins in clusters 3 and 4 can potentially be viewed as serum biomarkers or potentially candidates for therapeutics inhibition to mitigate fibrovascular scar healing in tendon.

This study has yielded novel insights into the transcriptional upstream regulators that drive enhanced tendon healing and fibrosis. However, there are limitations that qualify the conclusions of our study. First, our study was limited in assessing transcriptional regulation in bulk tendon tissue in native tendon and 7 days after injury. Whole-genome transcriptional profiling from the injured tendon tissue site represents a heterogeneous cell population of tenocytes, fibroblasts, myofibroblasts and immune cells, which could be resolved by single-cell RNA-sequencing in the tendon healing as recently described ${ }^{57}$. Future analysis will benefit from the utilization of single cell RNA-sequencing or spatial transcriptomics to elucidate signaling mechanisms at a cellular level given the involvement of the heterogeneous cellular populations at the tendon injury site and at later time-points that would represent the fibro-proliferative and remodeling phases of wound healing. Another limitation is that only male mice were included in this study. There are mixed reports on sex differences in the mechanical properties and biochemical composition of male and female tendons ${ }^{58}$ and in their response to injury $y^{59}$. With respect to incidence of flexor tendon lacerations in humans, males are 5:1 more likely to experience flexor tendon lacerations compared to females ${ }^{60}$, which justifies the focus on male animals in this study. However, future studies should evaluate potential sex differences flexor tendon healing in the C57 and MRL mice.

In summary, this study identified critical cell cycle regulation and differentiation roles for TGFB1 signaling that contribute to improved tendon repair following injury in MRL flexor tendons but not in the C57 tendons. Uncovering the unique cellular and molecular pathways in which TGFB1 induces regenerative tendon healing in the MRL model could help identify therapeutic targets for regenerative tendon healing.

\section{Materials and methods}

Animal care and flexor tendon surgery. The study is in accordance with relevant guidelines and regulations. All methods are reported in accordance with ARRIVE guidelines (https://arriveguidelines.org) ${ }^{61}$. All animal experimental protocols were approved by University of Rochester Committee on Animal Research (UCAR). Breeding pairs for the C57BL/6J (C57; stock number: 000664) and MRL/MpJ (MRL; stock number: 000486) were obtained from the Jackson Laboratory (Bar Harbor, ME) and bred in-house. Nine to eleven-week-old male mice from each strain were randomized into experimental groups. The mouse surgery protocol involves a partial laceration of the deep digital flexor (DDF) tendon of the 3rd digit in the hind paw, as previously described ${ }^{30}$. Surgeries were performed under a stereomicroscope on the hind paws using sterile aseptic techniques. Mice were anesthetized with $60 \mathrm{mg} / \mathrm{kg}$ ketamine and $4 \mathrm{mg} / \mathrm{kg}$ xylazine. A transverse incision was made on the 3rd digit between the metatarsophalangeal (MTP) and the proximal interphalangeal (PIP) joints. A transverse, mediolateral cut across roughly $50 \%$ of the DDF tendon width was completed. The skin incision was closed using interrupted sutures (Ethicon Suture, V950G, 9-0). To minimize pain, all mice were subcutaneously, Buprenorphine was administered immediately after closure of the skin incision $(0.05 \mathrm{mg} / \mathrm{kg})$ and postoperatively every $24 \mathrm{~h}$ up to $72 \mathrm{~h}$. Blood was collected from mice whose hind paws were used for gene expression analysis.

Biomechanical testing. Tensile elastic biomechanical testing was completed as previously described ${ }^{30}$. The DDFT was released at the myotendinous junction, precisely dissected along the tibia and tarsal bones, and released from the tarsal tunnel pulleys. The middle (3rd) tendon was isolated, with the tendon-to-bone attachment (enthesis) left intact for mounting and gripping. Tendons were loaded in a custom uniaxial microtester (eXpert 4000 MicroTester, ADMET, Inc., Norwood, MA) with the 3rd digit tip gripped between the two stainless steel plates. After preloading to $0.05 \mathrm{~N}$, tendon width, thickness, and gauge length were measured while the tissue was immersed in a $1 \times$ PBS bath and imaged on the microscope. Tendon gauge length was measured as the length of the tendon between the Microtester system clamps. Width measurements were taken first, then the tendon was rotated 90 degrees in the clamps to measure thickness. Width and thickness measurements were taken at 3-5 locations along the length of the tendon and averaged. Cross sectional area was assumed to be an ellipse. Based on each sample's total gauge length, each sample underwent uniaxial displacement-controlled biomechanical testing as follows; $1 \%$ strain preconditioning for 10-cycles, returned to initial gauge length to rest for $5 \mathrm{~min}$, stretched to $5 \%$ strain $(0.05 \mathrm{strain} / 8 \mathrm{~s})$ and held for $10 \mathrm{~min}$ to allow for stress-relaxation, returned to initial gauge length to rest for $5 \mathrm{~min}$, and concluded with a displacement-controlled ramp to failure at $0.05 \mathrm{~mm} /$ $\mathrm{sec}$. Structural and material properties were determined from the ramp to failure force-displacement and stressstrain curves, respectively. All mechanical testing analysis was completed with a custom Matlab (MathWorks, Natick, MA) code. Sample size of $\mathrm{N}=4-6$ for each mouse strain and each experimental timepoint.

Histology and immunohistochemistry. The hind paw was transected near the tarsal bones and prepared for histology by scoring the skin superficial to the footpad to enhance 10\% Neutral Buffered Formalin (NBF) tissue infiltration. Tissues were fixed in $10 \% \mathrm{NBF}$ for $48 \mathrm{~h}$ at room temperature (RT), followed by decalcification in calis-EDTA ( $\mathrm{pH} 7.2-7.4$ ) for 7 days at RT. The tissues were then dehydrated in an ethanol gradient and embedded in paraffin. Paraffin-embedded tissues were cut axially into $5 \mu \mathrm{m}$ thick sections between the metatarsophalangeal (MTP) joint and the A3 pulley of each sample, then stained for $15 \mathrm{~s}$ with Mayer's Hematoxylin. Sections for histomorphometric analysis were immediately cover slipped with a water-based mounting medium (Faramount Aqueous Mounting Medium, Dako, \#S3025), whereas sections for immunohistochemistry (IHC) were cover slipped after IHC. 
For immunohistochemistry, paraffin-embedded and sectioned tissues were collected at $0,14,28$, and 56 days

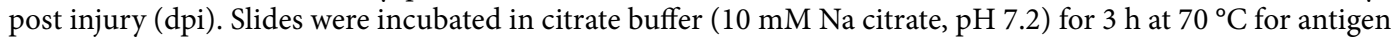
retrieval, circled with a hydrophobic barrier pen (Vector Laboratories, Burlingame, CA), and blocked with an endogenous peroxidase/ alkaline phosphatase inhibitor (Bloxall, Vector Laboratories). After washing with PBST (PBS+ $0.1 \%$ Tween-20), they were incubated for $1 \mathrm{~h}$ at room temperature in blocking solution (5\% normal horse serum+ PBST). Sections were incubated for $1 \mathrm{~h}$ at RT with the primary antibody mouse monoclonal PCNA $(\mathrm{ab} 29, \mathrm{Abcam})$ at 1:1000 $(1 \mu \mathrm{g} / \mathrm{mL})$ dilution. Followed by Vectastain Elite Kit with DAB chromogen (Vector Laboratories). Immunohistochemical sections were rinsed with deionized water, counterstained with Mayer's Hematoxylin, cover slipped with a water-based mounting medium (Faramount Aqueous Mounting Medium, Dako, \#S3025), and imaged with a VS120 Virtual Slide Microscope (Olympus, Waltham, MA).

Quantification of histomorphometric and immunohistochemical images. For histomorphometry, paraffin-embedded tissues were prepared as described above. Brightfield images were obtained from the slide scanner and processed using Visiopharm image analysis software v.6.7.0.2590 (Visiopharm, Hørsholm, Denmark). Regions of interest were manually drawn to define the tendon tissue area inside the synovial sheath. Using a custom Visiopharm program, imaged sections were Bayesian classified and thresholded to train the program to specific pixel RGB colors and intensities. To improve accuracy and repeatability, post-processing analyses defined cell nuclei area $>2 \mu \mathrm{m}^{2}$, synovial sheath space (SS) $5 \mu \mathrm{m}^{2}<\mathrm{SS}<5000 \mu \mathrm{m}^{2}$, eroded local minima pixel intensities for each colorimetric label, and labeled blank space within the tendon body as intratendinous space. Magnified brightfield images of hematoxylin stained section $(10 \times, 20 \times)$ were manually delineated to distinguish the superficial digital flexor tendons (SDFT) from the deep digital flexor tendons (DDFT). The following tissue measurements were taken: DDF tendon cross-sectional area, synovial sheath space (SS), tendon extracellular matrix (ECM), intratendinous cell nuclei count, intratendinous cellular density, and length of DDFT perimeter adhered to subcutaneous tissues ${ }^{30}$. Each histomorphometric outcome measure was quantified using Visiopharm software. Quantitative histomorphometric scoring was averaged for $\mathrm{N}=4-5$ animals per experimental group $(n=3-5$ images (technical replicates) per animal). Additional plantar 3 micron-thick sections of the injured tendons were stained with Masson's Trichrome to assess collagen deposition. For immunofluorescent staining, sections were stained with F4/80 (1:50, Cat\#: ab6640), Bcl2 (1:500, Cat\#: ab182858), a-SMA-FITC (1:200, Cat\#: F3777), or Histone H2A.X (phospho S139) (1:100, Cat\#: 05-636). Sections were counterstained with a DAPI nuclear stain and imaged with a VS120 Virtual Slide Microscope (Olympus).

RNA extraction. Tendon tissue was collected from the injury site for RNA extraction and subsequent gene expression analysis by the URMC Genomics Core. Briefly, partially lacerated, or uninjured tendon tissue was flash frozen in liquid nitrogen and stored at $-80{ }^{\circ} \mathrm{C}$ until extraction. Peritendinous tissue and surrounding sheath tissue were not removed due to size limitations. Total RNA was isolated from single tendons utilizing Trizol (ThermoFisher, \#15596026) extraction methods and a bullet blender for tissue homogenization. RNA concentration was determined with the NanoDrop 1000 spectrophotometer (NanoDrop, Wilmington, DE) and RNA quality assessed with the Agilent Bioanalyzer 2100 (Agilent, Santa Clara, CA).

Next-generation sequencing (NGS) data processing and alignment. Samples of 1 ng of total RNA were pre-amplified with the SMARTer Ultra Low Input kit v4 (Clontech, Mountain View, CA) per manufacturer's recommendations. The quantity and quality of the subsequent cDNA was determined using the Qubit Flourometer (Life Technnologies, Carlsbad, CA) and the Agilent Bioanalyzer 2100 (Agilent, Santa Clara, CA). $150 \mathrm{pg}$ of cDNA was used to generate Illumina compatible sequencing libraries with the NexteraXT library preparation kit (Illumina, San Diego, CA) per manufacturer's protocols. The amplified libraries were hybridized to the Illumina single end flow cell and amplified using the cBot (Illumina, San Diego, CA). Single end reads of $100 \mathrm{nt}$ were generated for each sample. Raw reads generated from the Illumina HiSeq. 2500 sequencer were demultiplexed using bcl2fastq version 2.19.0. Quality filtering and adapter removal are performed using Trimmomatic version 0.36 with the following parameters: "TRAILING:13 LEADING:13 ILLUMINACLIP:adapters. fasta:2:30:10 SLIDINGWINDOW:4:20 MINLEN:15”. Quality reads were then mapped to the Mus musculus reference sequence (GRCm38.p5) with STAR_2.5.2b with the following parameters: "--twopassMode Basic --runMode alignReads --genomeDir $\$\{$ GENOME $\}$--readFilesIn $\$\{$ SAMPLE $\}$--outSAMtype BAM SortedByCoordinate --outSAMstrandField intronMotif --outFilterIntronMotifs RemoveNoncanonical". Differential expression analysis and data normalization were performed using default methods in DESeq. 2-1.14.1 with an adjusted $p$ value threshold of 0.05 .

Bioinformatics and statistical analyses of DEG. Differential expression analysis and data normalization were performed using DESeq. 2-1.14.1 on RNA extracted from injured tendons at 7 days post injury (dpi) relative to uninjured expression levels within each strain independently ( $\mathrm{N}=4$ animals per experimental group). DEG was identified by filtering DeSeq. 2 pairwise comparisons for biological (ABS(Log2FC) $>1$ ) and statistical significance (adjusted $p<0.05$ ). Functional enrichment of upstream regulators was completed in pathway enrichment analysis using Ingenuity Pathway Analysis (IPA; http://www.ingenuity.com). Default settings were used to determine upstream regulators using the global molecular network contained in the IPA knowledge base. Downstream targets of the upstream regulator TGF- $\beta 1$ identified in IPA were exported for further functional analysis using Enrichr (https://maayanlab.cloud/Enrichr/) and Reactome (https://reactome.org).

Multiplex serum protein assay. Peripheral blood was collected via submandibular bleeds $(\sim 0.2-0.5 \mathrm{ml})$ from uninjured and at $1,3,5,7$, and 14 days post injury (dpi). The blood clotted completely at room tempera- 
ture, was centrifuged to isolate blood serum from coagulate, then the blood serum $(\sim 20-40 \mu \mathrm{l})$ was transferred to cryovials for storage at $-80{ }^{\circ} \mathrm{C}$ before shipping to EVE Technologies for multiplex protein analysis (EVE Technologies 3415A-3 Ave., N.W. Calgary, AB Canada T2N 0M4). Serum protein analysis was performed with three analytical arrays of cytokines, chemokines, and transforming growth factor beta isoforms. Sample size was 3 mice per strain per time-point. Serum protein concentrations for Day 5 C57 contain only two samples because the third serum sample is a statistical outlier. We used the Prism ROUT outlier detection test $(\mathrm{Q}=1 \%)^{62}$, which confirmed that these measurements were statistical outliers, and were therefore removed from the analysis.

Statistical analyses. All histomorphometry and biomechanics data were graphed and statistically analyzed with GraphPad Prism (GraphPad Software). All data were tested for normality using the Kolmogorov-Smirnov test in Prism before conducting statistical comparisons that assume normal distributions (e.g. ANOVA). Significant differences $(p<0.05)$ for all data were determined using a 2-way ANOVA and Bonferroni-corrected multiple comparison post-tests. Differential expressional analysis and plots was generated in R Studio with an adjusted $p$-value threshold of 0.05 .

\section{Data availability}

The RNA-seq raw and processed data were deposited in the Gene Expression Omnibus(GEO) under accession GSE175912.

Received: 3 June 2021; Accepted: 11 February 2022

Published online: 22 February 2022

\section{References}

1. Pryce, B. A. et al. Recruitment and maintenance of tendon progenitors by TGFbeta signaling are essential for tendon formation. Development 136, 1351-1361. https://doi.org/10.1242/dev.027342 (2009).

2. Schweitzer, R. et al. Analysis of the tendon cell fate using Scleraxis, a specific marker for tendons and ligaments. Development 128, 3855-3866 (2001).

3. Berthet, E. et al. Smad3 binds Scleraxis and Mohawk and regulates tendon matrix organization. J. Orthop. Res. 31, 1475-1483. https://doi.org/10.1002/jor.22382 (2013).

4. Ito, Y. et al. The Mohawk homeobox gene is a critical regulator of tendon differentiation. Proc. Natl. Acad. Sci. USA 107, 1053810542. https://doi.org/10.1073/pnas.1000525107 (2010).

5. Alberton, P. et al. Loss of tenomodulin results in reduced self-renewal and augmented senescence of tendon stem/progenitor cells. Stem Cells Dev. 24, 597-609. https://doi.org/10.1089/scd.2014.0314 (2015).

6. Dex, S. et al. Tenomodulin is required for tendon endurance running and collagen I Fibril adaptation to mechanical load. EBioMedicine 20, 240-254. https://doi.org/10.1016/j.ebiom.2017.05.003 (2017).

7. Whalen, W. P. Utilization of scar tissue in bridging tendon defects. Ann. Surg. 133, 567-571. https://doi.org/10.1097/00000658195104000-00019 (1951).

8. Tempfer, H. \& Traweger, A. Tendon vasculature in health and disease. Front. Physiol. 6, 330. https://doi.org/10.3389/fphys.2015. 00330 (2015).

9. Andarawis-Puri, N. \& Flatow, E. L. Promoting effective tendon healing and remodeling. J. Orthop. Res. 36, 3115-3124. https://doi. org/10.1002/jor.24133 (2018).

10. Thomopoulos, S., Parks, W. C., Rifkin, D. B. \& Derwin, K. A. Mechanisms of tendon injury and repair. J. Orthop. Res. 33, 832-839. https://doi.org/10.1002/jor.22806 (2015).

11. Dyment, N. A. \& Galloway, J. L. Regenerative biology of tendon: mechanisms for renewal and repair. Curr. Mol. Biol. Rep. 1, 124-131. https://doi.org/10.1007/s40610-015-0021-3(2015).

12. Kaji, D. A., Howell, K. L., Balic, Z., Hubmacher, D. \& Huang, A. H. Tgfbeta signaling is required for tenocyte recruitment and functional neonatal tendon regeneration. Elife https://doi.org/10.7554/eLife.51779 (2020).

13. Howell, K. et al. Novel model of tendon regeneration reveals distinct cell mechanisms underlying regenerative and fibrotic tendon healing. Sci. Rep. 7, 45238. https://doi.org/10.1038/srep45238 (2017).

14. Dyment, N. A. et al. The paratenon contributes to scleraxis-expressing cells during patellar tendon healing. PLoS ONE 8, e59944. https://doi.org/10.1371/journal.pone.0059944 (2013).

15. Best, K. T. \& Loiselle, A. E. Scleraxis lineage cells contribute to organized bridging tissue during tendon healing and identify a subpopulation of resident tendon cells. FASEB J. 33, 8578-8587. https://doi.org/10.1096/f.201900130RR (2019).

16. Nichols, A. E. C., Best, K. T. \& Loiselle, A. E. The cellular basis of fibrotic tendon healing: challenges and opportunities. Transl. Res. 209, 156-168. https://doi.org/10.1016/j.trsl.2019.02.002 (2019).

17. Clark, L. D., Clark, R. K. \& Heber-Katz, E. A new murine model for mammalian wound repair and regeneration. Clin. Immunol. Immunopathol. 88, 35-45. https://doi.org/10.1006/clin.1998.4519 (1998).

18. Beare, A. H., Metcalfe, A. D. \& Ferguson, M. W. Location of injury influences the mechanisms of both regeneration and repair within the MRL/MpJ mouse. J. Anat. 209, 547-559. https://doi.org/10.1111/j.1469-7580.2006.00641.x (2006).

19. Heydemann, A. et al. The superhealing MRL background improves muscular dystrophy. Skelet. Muscle 2, 26. https://doi.org/10. 1186/2044-5040-2-26 (2012).

20. Tseng, C. et al. Markers of accelerated skeletal muscle regenerative response in murphy Roths large mice: Characteristics of muscle progenitor cells and circulating factors. Stem Cells 37, 357-367. https://doi.org/10.1002/stem.2957 (2019).

21. Fitzgerald, J. Enhanced cartilage repair in "healer" mice-New leads in the search for better clinical options for cartilage repair. Semin. Cell Dev. Biol. 62, 78-85. https://doi.org/10.1016/j.semcdb.2016.04.018 (2017).

22. Leonard, C. A. et al. Allogeneic bone marrow transplant from MRL/MpJ super-healer mice does not improve articular cartilage repair in the C57Bl/6 strain. PLoS ONE 10, e0131661. https://doi.org/10.1371/journal.pone.0131661 (2015).

23. Rai, M. F. \& Sandell, L. J. Regeneration of articular cartilage in healer and non-healer mice. Matrix Biol. 39, 50-55. https://doi.org/ 10.1016/j.matbio.2014.08.011 (2014).

24. George, N. S., Bell, R., Paredes, J. J., Taub, P. J. \& Andarawis-Puri, N. Superior mechanical recovery in male and female MRL/MpJ tendons is associated with a unique genetic profile. J. Orthop. Res. https://doi.org/10.1002/jor.24705 (2020).

25. Lalley, A. L. et al. Improved biomechanical and biological outcomes in the MRL/MpJ murine strain following a full-length patellar tendon injury. J. Orthop. Res. 33, 1693-1703. https://doi.org/10.1002/jor.22928 (2015).

26. Paredes, J., Marvin, J. C., Vaughn, B. \& Andarawis-Puri, N. Innate tissue properties drive improved tendon healing in MRL/MpJ and harness cues that enhance behavior of canonical healing cells. FASEB J. 34, 8341-8356. https://doi.org/10.1096/fj.201902825RR (2020). 
27. Davis, T. A., Longcor, J. D., Hicok, K. C. \& Lennon, G. G. Prior injury accelerates subsequent wound closure in a mouse model of regeneration. Cell Tissue Res. 320, 417-426. https://doi.org/10.1007/s00441-005-1107-7 (2005).

28. Sebastian, A. et al. Comparative transcriptomics identifies novel genes and pathways involved in post-traumatic osteoarthritis development and progression. Int. J. Mol. Sci. https://doi.org/10.3390/ijms19092657 (2018).

29. Caldwell, R. L., Opalenik, S. R., Davidson, J. M., Caprioli, R. M. \& Nanney, L. B. Tissue profiling MALDI mass spectrometry reveals prominent calcium-binding proteins in the proteome of regenerative MRL mouse wounds. Wound Repair. Regen. 16, 442-449. https://doi.org/10.1111/j.1524-475X.2007.00351.x (2008).

30. Freeberg, M. A. T. et al. Serpine1 knockdown enhances MMP activity after flexor tendon injury in mice: Implications for adhesions therapy. Sci. Rep. 8, 5810. https://doi.org/10.1038/s41598-018-24144-1 (2018).

31. Best, K. T. et al. NF-kB activation persists into the remodeling phase of tendon healing and promotes myofibroblast survival. Sci. Signal https://doi.org/10.1126/scisignal.abb7209 (2020).

32. Elliot, D., Lalonde, D. H. \& Tang, J. B. Commentaries on Clinical results of releasing the entire A2 pulley after flexor tendon repair in zone 2C. K. Moriya, T. Yoshizu, N. Tsubokawa, H. Narisawa, K. Hara and Y. Maki. J Hand Surg Eur. 2016, 41: 822-28. J. Hand. Surg. Eur. Vol. 41, 829-830, https://doi.org/10.1177/1753193416655932 (2016).

33. Tang, J. B. et al. Current practice of primary flexor tendon repair: a global view. Hand Clin. 29, 179-189. https://doi.org/10.1016/j. hcl.2013.02.003 (2013)

34. Zhou, X., Li, X. R., Qing, J., Jia, X. F. \& Chen, J. Outcomes of the six-strand M-Tang repair for zone 2 primary flexor tendon repair in 54 fingers. J. Hand Surg. Eur. https://doi.org/10.1177/1753193417691390 (2017).

35. Freeberg, M. A. T. et al. Transcriptomic analysis of cellular pathways in healing flexor tendons of plasminogen activator inhibitor 1 (PAI-1/Serpine1) null mice. J. Orthop. Res. https://doi.org/10.1002/jor.24448 (2019).

36. Paredes, J., Shiovitz, D. A. \& Andarawis-Puri, N. Uncorrelated healing response of tendon and ear injuries in MRL highlight a role for the local tendon environment in driving scarless healing. Connect Tissue Res. 59, 472-482. https://doi.org/10.1080/03008207. 2018.1485665 (2018).

37. Wojciak, B. \& Crossan, J. F. The effects of T cells and their products on in vitro healing of epitenon cell microwounds. Immunology 83, 93-98 (1994).

38. Wang, S. \& Hirschberg, R. BMP7 antagonizes TGF-beta -dependent fibrogenesis in mesangial cells. Am. J.Physiol. Renal. Physiol. 284, F1006-1013. https://doi.org/10.1152/ajprenal.00382.2002 (2003).

39. Chang, J. et al. Gene expression of transforming growth factor beta-1 in rabbit zone II flexor tendon wound healing: evidence for dual mechanisms of repair. Plast. Reconstr. Surg. 100, 937-944. https://doi.org/10.1097/00006534-199709001-00016 (1997).

40. Beredjiklian, P. K. et al. Regenerative versus reparative healing in tendon: A study of biomechanical and histological properties in fetal sheep. Ann. Biomed. Eng. 31, 1143-1152. https://doi.org/10.1114/1.1616931 (2003).

41. Krummel, T. M. et al. Transforming growth factor beta (TGF-beta) induces fibrosis in a fetal wound model. J. Pediatr. Surg. 23, 647-652. https://doi.org/10.1016/s0022-3468(88)80638-9 (1988).

42. Lebrun, J. J. The dual role of TGFbeta in human cancer: From tumor suppression to cancer metastasis. ISRN Mol. Biol. 2012, 381428. https://doi.org/10.5402/2012/381428 (2012).

43. Saxena, V. et al. Dual roles of immunoregulatory cytokine TGF-beta in the pathogenesis of autoimmunity-mediated organ damage. J. Immunol. 180, 1903-1912. https://doi.org/10.4049/jimmunol.180.3.1903 (2008).

44. Meng, X.-M. et al. Inflammatory macrophages can transdifferentiate into myofibroblasts during renal fibrosis. Cell Death Dis. 7, e2495-e2495. https://doi.org/10.1038/cddis.2016.402 (2016).

45. Wang, S. et al. TGF- $\beta / \mathrm{Smad} 3$ signalling regulates the transition of bone marrow-derived macrophages into myofibroblasts during tissue fibrosis. Oncotarget 7, 8809-8822. https://doi.org/10.18632/oncotarget.6604 (2016).

46. Tchkonia, T., Zhu, Y., van Deursen, J., Campisi, J. \& Kirkland, J. L. Cellular senescence and the senescent secretory phenotype: therapeutic opportunities. J. Clin. Invest. 123, 966-972. https://doi.org/10.1172/JCI64098 (2013).

47. Shetty, S. K. et al. p53 and miR-34a feedback promotes lung epithelial injury and pulmonary fibrosis. Am. J. Pathol. 187, 1016-1034. https://doi.org/10.1016/j.ajpath.2016.12.020 (2017).

48. Higgins, S. P. et al. TGF-beta1/p53 signaling in renal fibrogenesis. Cell Signal 43, 1-10. https://doi.org/10.1016/j.cellsig.2017.11. $005(2018)$

49. Ruwanpura, S. M., Thomas, B. J. \& Bardin, P. G. Pirfenidone: Molecular mechanisms and potential clinical applications in lung disease. Am. J. Respir. Cell Mol. Biol. 62, 413-422. https://doi.org/10.1165/rcmb.2019-0328TR (2020).

50. Usugi, E. et al. Antifibrotic agent pirfenidone suppresses proliferation of human pancreatic cancer cells by inducing G0/G1 cell cycle arrest. Pharmacology 103, 250-256. https://doi.org/10.1159/000496831 (2019).

51. Jablonski, C. L., Besler, B. A., Ali, J. \& Krawetz, R. J. p21(-/-) mice exhibit spontaneous articular cartilage regeneration post-injury. Cartilage https://doi.org/10.1177/1947603519876348 (2019).

52. Bedelbaeva, K. et al. Lack of p21 expression links cell cycle control and appendage regeneration in mice. Proc. Natl. Acad. Sci. USA 107, 5845-5850. https://doi.org/10.1073/pnas.1000830107 (2010).

53. Naviaux, R. K. et al. Retained features of embryonic metabolism in the adult MRL mouse. Mol. Genet. Metab. 96, 133-144. https:// doi.org/10.1016/j.ymgme.2008.11.164 (2009)

54. Ferguson, M. W. \& O'Kane, S. Scar-free healing: from embryonic mechanisms to adult therapeutic intervention. Philos. Trans. R. Soc. Lond. B Biol. Sci. 359, 839-850. https://doi.org/10.1098/rstb.2004.1475 (2004).

55. Nukui, Y. et al. Serum CXCL9 and CCL17 as biomarkers of declining pulmonary function in chronic bird-related hypersensitivity pneumonitis. PLoS ONE 14, e0220462. https://doi.org/10.1371/journal.pone.0220462 (2019).

56. Sahin, H. et al. Chemokine Cxcl9 attenuates liver fibrosis-associated angiogenesis in mice. Hepatology 55, 1610-1619. https://doi. org/10.1002/hep.25545 (2012).

57. Harvey, T., Flamenco, S. \& Fan, C. M. A Tppp3(+)Pdgfra(+) tendon stem cell population contributes to regeneration and reveals a shared role for PDGF signalling in regeneration and fibrosis. Nat. Cell Biol. 21, 1490-1503. https://doi.org/10.1038/s41556-0190417-z (2019).

58. Sarver, D. C. et al. Sex differences in tendon structure and function. J. Orthop. Res. 35, 2117-2126. https://doi.org/10.1002/jor. 23516 (2017).

59. Silbernagel, K. G. et al. Sex differences in outcome after an acute achilles tendon rupture. Orthop. J. Sports Med. 3 , 2325967115586768. https://doi.org/10.1177/2325967115586768 (2015).

60. de Jong, J. P. et al. The incidence of acute traumatic tendon injuries in the hand and wrist: a 10-year population-based study. Clin Orthop Surg 6, 196-202. https://doi.org/10.4055/cios.2014.6.2.196 (2014).

61. Kilkenny, C., Browne, W. J., Cuthill, I. C., Emerson, M. \& Altman, D. G. Improving bioscience research reporting: The ARRIVE guidelines for reporting animal research. PLoS Biol 8, e1000412. https://doi.org/10.1371/journal.pbio.1000412 (2010).

62. Motulsky, H. J. \& Brown, R. E. Detecting outliers when fitting data with nonlinear regression-A new method based on robust nonlinear regression and the false discovery rate. BMC Bioinformatics 7, 123. https://doi.org/10.1186/1471-2105-7-123 (2006).

\section{Acknowledgements}

The study was supported by grant numbers R01AR056696, R01AR073169, UG3TR003281, and P30AR069655 from NIAMS/NIH. MTF was supported by the Training In Orthopaedic Research Program funded by NIH 
T32AR053459. The content is solely the responsibility of the authors and does not necessarily represent the official views of the National Institute of General Medical Sciences or NIH. We thank Brittany Strauss, Sarah Mack, Jeff Fox, Kathleen Maltby for histology assistance.

\section{Author contributions}

Conceptualization: H.A.A., D.A., J.G.K., M.T.F., A.W. Methodology: J.G.K., D.A., M.T.F., R.E.A., R.G.A., S.M., J.A., M.R.B., A.L. Software: J.G.K., M.T.F., J.M. Formal Analysis: J.G.K., M.T.F., D.A., R.G.A., M.R.B., J.A., J.M., A.V.W., H.A.A. Investigation: J.G.K., D.A., M.T.F. Writing-Original Draft: J.G.K., M.T.F., H.A.A. WritingReviewing and Editing: J.G.K., M.T.F., A.L., R.E.A., R.G.A., S.M., M.B., A.W., H.A.A. Visualization: J.G.K., M.T.F., R.G.A., H.A.A. Supervision: H.A. Project Administration: H.A.A. Funding Acquisition: H.A.A. All authors reviewed the manuscript before submission.

\section{Competing interests}

The authors declare no competing interests.

\section{Additional information}

Supplementary Information The online version contains supplementary material available at https://doi.org/ 10.1038/s41598-022-07124-4.

Correspondence and requests for materials should be addressed to H.A.A.

Reprints and permissions information is available at www.nature.com/reprints.

Publisher's note Springer Nature remains neutral with regard to jurisdictional claims in published maps and institutional affiliations.

(c) (i) Open Access This article is licensed under a Creative Commons Attribution 4.0 International License, which permits use, sharing, adaptation, distribution and reproduction in any medium or format, as long as you give appropriate credit to the original author(s) and the source, provide a link to the Creative Commons licence, and indicate if changes were made. The images or other third party material in this article are included in the article's Creative Commons licence, unless indicated otherwise in a credit line to the material. If material is not included in the article's Creative Commons licence and your intended use is not permitted by statutory regulation or exceeds the permitted use, you will need to obtain permission directly from the copyright holder. To view a copy of this licence, visit http://creativecommons.org/licenses/by/4.0/.

(C) The Author(s) 2022 